\title{
QUEEN'S
UNIVERSITY
BELFAST
}

\section{Polarisation independent resistively loaded frequency selective surface absorber with optimum oblique incidence performance}

Zabri, N., Cahill, R., \& Schuchinsky, A. (2014). Polarisation independent resistively loaded frequency selective surface absorber with optimum oblique incidence performance. IET Microwaves, Antennas and Propagation, 8(14), 1198-1203. https://doi.org/10.1049/iet-map.2014.0124

\section{Published in:}

IET Microwaves, Antennas and Propagation

\section{Document Version:}

Peer reviewed version

Queen's University Belfast - Research Portal:

Link to publication record in Queen's University Belfast Research Portal

\section{Publisher rights}

(c) The Institution of Engineering and Technology 2014

This paper is a postprint of a paper submitted to and accepted for publication in IET Microwaves Antennas and Propagation and is subject to Institution of Engineering and Technology Copyright. The copy of record is available at IET Digital Library

\section{General rights}

Copyright for the publications made accessible via the Queen's University Belfast Research Portal is retained by the author(s) and / or other copyright owners and it is a condition of accessing these publications that users recognise and abide by the legal requirements associated with these rights.

Take down policy

The Research Portal is Queen's institutional repository that provides access to Queen's research output. Every effort has been made to ensure that content in the Research Portal does not infringe any person's rights, or applicable UK laws. If you discover content in the Research Portal that you believe breaches copyright or violates any law, please contact openaccess@qub.ac.uk. 


\title{
Polarization Independent Resistively Loaded FSS Absorber with Optimum Oblique Incidence Performance
}

\author{
Normi Zabri, Robert Cahill and Alexander Schuchinsky \\ The Institute of Electronics, Communications and Information Technology, \\ Queen's University Belfast, Northern Ireland Science Park, Queen's Road, Queen's Island, \\ Belfast, BT3 9DT, Northern Ireland, UK \\ Email: r.cahill@qub.ac.uk
}

Key Words: Frequency Selective Surfaces (FSS), electromagnetic wave absorption, radar absorbing material (RAM), artificial magnetic conductor (AMC), high impedance surfaces (HIS)

\section{ABSTRACT}

This paper presents the design of a thin electromagnetic absorber which exhibits radar backscatter suppression that is independent of the wave polarization at large incidence angles. The structure consists of a metal backed printed Frequency Selective Surface (FSS), with resistors placed across narrow gaps inserted in the middle of each of the four sides of the conductor loops. The geometry of the periodic array and the value of the vertical and horizontal resistor pairs are carefully chosen to present a real impedance of $377 \Omega$ at the center operating frequency for both TE and TM polarized waves. Angular sensitivity and reflectivity bandwidth have been investigated for FSS absorber designs with thickness 1,2 and $3 \mathrm{~mm}$. Each of the three structures was optimized to work at a center frequency of $10 \mathrm{GHz}$ and an incident angle of $45^{\circ}$. The design methodology is verified by measuring the radar backscatter suppression from a $3 \mathrm{~mm}(\lambda / 10)$ thick screen in the frequency range $8-12$ $\mathrm{GHz}$. The absorber construction was simplified by filling the four metal gaps in each unit cell with shielding paint, and selecting the ink thickness to give the two required surface resistance values. 


\section{INTRODUCTION}

Classical microwave absorbers such as the $\lambda / 4$ thick Salisbury screen are simple to design and construct [1], [2], and therefore are widely used to suppress electromagnetic energy scattered from metal objects. However these structures cannot be used to provide radar cloaking of wind turbines and vehicular platforms where thickness and weight are key design drivers. For these applications and others which require narrow band operation, the deployment of ultra-thin microwave absorbers based on resistively loaded high impedance surfaces is an attractive solution. The analysis and design of thin FSS absorbers is generally based on symmetrical periodic elements such as square loops, patches and crossed dipoles [3], [4], [5]. The scattering behavior of these structures is identical in the TE and TM plane, but only at normal and small angles of incidence. Recently, there has been significant interest in developing frequency selective surfaces which exhibit similar spectral transmission responses when exposed to TE and TM polarized signals at $45^{\circ}$ incidence [6], [7]. These structures are composed of nested short-circuited ( $\lambda$ and $\lambda / 2$ mode) annular slots which yield identical resonant frequencies in the two field directions, but the transmission bandwidth in the TM plane is always significantly narrower. The authors have demonstrated that this performance limitation can be removed in reflection mode, by printing two different ( $\lambda$ mode) split ring arrays on the opposite sides of a thin dielectric substrate, one of which provides the TE and the other the TM response, [8]. In this paper we build on the work reported in [8] to create a thin metal backed FSS absorber which provides significant suppression of the backscattered signals at oblique incidence with the electric vectors orientated either vertically (TE) or horizontally (TM). Independent control of the reflectivity in the two planes of polarization, and equalisation of the bandwidth and resonant frequencies is achieved by using a split ring resonator [8] composed of a single periodic array of rectangular loops with resistive elements inserted at the midpoint of the four sides. Proof of the principle of operation is made by designing an absorber which functions for both polarisations when tilted $45^{\circ}$ with respect to the angle of incidence. For this orientation, far from normal incidence, it 
very challenging to create the in plane anisotropy that is required to balance TE and TM waves [6], [7], [8]. Numerical predictions are employed to optimize the geometry of the structure, obtain the value of the resistor pairs and study the dependence of reflectivity bandwidth and angular sensitivity on the absorber thickness. An experimental prototype structure was constructed using different thicknesses of high frequency shielding paint to realise the two surface resistance values required for the resistor pairs. Bistatic measurements of the radar backscatter at large incidence angles are shown to be in good agreement with the computed results over the frequency range $8-12 \mathrm{GHz}$.

\section{THEORY OF OPERATION}

The equivalent circuit of a thin high impedance surface (HIS) consists of a parallel connection of the FSS impedance which can be represented by a series L, C, R circuit, and the transformed impedance, $L^{\prime}$, of the metal backing plate behind the periodic array. Resonance occurs at the frequency where the imaginary parts of the FSS impedance and the inductance presented by the ground plane cancel each other. By selecting the value of $R$, which is used to model the loss of the FSS array, it is possible to impedance match the structure to free space $(377 \Omega)$ thereby creating a 'perfect' absorber at resonance. At normal incidence the impedance of the HIS is independent of the orientation of the incident waves for FSS constructed of symmetrical periodic elements. However at oblique incidence the impedance is strongly dependent on the angle and plane of polarization [9], therefore the absorption band shifts to different center frequencies in the TE and TM plane and a significant narrowing of the reflectivity bandwidth is observed for the latter electric field orientation.

Polarization independent suppression of the radar backscatter at large incident angles therefore requires an arrangement with either resistively loaded asymmetric [10] or nested FSS elements [6], [7] to yield coincident reflection responses. The latter class of FSS employs two elements in each unit cell, whereas the former option is simpler to design because independent and controllable spectral responses can be obtained in the TE and TM plane using just one periodic element. An 
example of this simpler arrangement is shown in [10], where an array of crossed dipoles with unequal arm lengths and conductor widths is shown to yield coincident spectral transmission responses at $45^{\circ}$ incidence. However this approach cannot be used to create the conductive periodic array of an HIS microwave absorber because the value of the resistor would be same for the two wave polarizations when placed at the current peak which is located at the center of the dipole arms. To overcome this problem and design a microwave absorber with a single periodic array, we have introduced a rectangular loop element which is resistively loaded across the gaps at the center of the four sides as depicted in Figure 1. When the structure is illuminated by a vertically polarized wave, the elements resonate in a $(\lambda)$ mode with a current null at position ' $A$ ' [11] (therefore the impact of $R_{T M}$ is negligible) and a current peak at ' $B$ ' the location of the horizontal resistor pairs $\left(R_{T E}\right)$. For a TM polarized signal the positions of the current nulls and peaks are interchanged, therefore for each electric vector direction, the induced sinusoidal current is affected by either the vertical or the horizontal resistor pairs, but not both.

\section{NUMERICAL OPTIMIZATION AND DESIGN}

CST Microwave Studio software [12] was employed to optimize the design, obtain the physical dimensions and compute the radar backscatter from the thin planar metal backed FSS absorber. By applying appropriate boundary conditions a single 3D unit cell was used to model the periodic structure that is infinite in extent. The unit cell arrangement shown in Figure 1 consists of an FSS screen printed on a $0.13 \mathrm{~mm}$ thick substrate with permittivity 2.2 and loss tangent 0.0009 (Taconic TLP-5) and separated from the ground plane by a practically lossless foam spacer with permittivity 1.05 (Rhoacell) and thickness $t_{a}$. For this study the $-10 \mathrm{~dB}$ reflectivity bandwidth and sensitivity to angle of incidence were investigated for structures with $t_{a}=1,2$ and $3 \mathrm{~mm}$. Each of the three absorbers was designed to work at $45^{\circ}$ incidence and exhibit the maximum obtainable reflectivity 
bandwidth centered at $10 \mathrm{GHz}$ for the two wave polarizations. The design process includes the following key stages

1) the resonances (reflection nulls) at the same frequency for TE and TM incidence were achieved by adjusting the length of the vertical and horizontal side arms of the printed rectangular loops.

2) the reflectivity bandwidth was maximized by employing a closely packed array of periodic elements with very wide conductors.

3) the depth of the reflectivity null at resonance was maximized by careful choice of the resistance values of the horizontal and vertical resistor pairs.

Figure 1 depicts the physical dimensions of the unit cell and resistor pair values for all three absorbers. The predicted reflection losses are compared at $45^{\circ}$ in Figure 2 where the suppression of the radar backscatter is shown to be more than $-40 \mathrm{~dB}$ at $10 \mathrm{GHz}$, the resonant frequency of the FSS absorber. Moreover the reflectivity plots in the TE and TM planes are virtually identical and the bandwidths are shown to be proportional to the absorber thickness. This well-known feature of HIS and reflectarray antenna [13] architectures is attributed to the variation in the inductance exhibited by the metal backing plate at the FSS reference plane. At $45^{\circ}$ incidence the $-10 \mathrm{~dB}$ reflectivity bandwidths for TE/TM are predicted to be $6 \%(1 \mathrm{~mm}-\lambda / 30), 16 \%(2 \mathrm{~mm}-\lambda / 15)$ and $36 \%(3 \mathrm{~mm}$ $\lambda / 10$ ) and the figure of merit (FOM) defined as the common (TE/TM) bandwidth divided by the physical thickness normalized to the center working frequency is 180, 240 and 360 respectively. These FOM values are in general smaller than FSS based absorbers designed to operate only at normal incidence [3], [5], however the $3 \mathrm{~mm}$ thick structure exceeds the 308 value for the classical Salisbury screen [1], [2].

Angular sensitivity is an important performance consideration when the absorber is required to work over a range of angles centered at $45^{\circ}$. We have investigated the angular sensitivity for each of the three structures by computing the reflectivity plots at $40^{\circ}$ and $50^{\circ}$. The results in Figure 3 show that 
in some cases there is a measurable narrowing of the absorption band and a shift in the resonant frequency which is much larger for TM polarized waves. The maximum reduction in the reflectivity bandwidth for the three cases plotted in Figure 4 is $1 \%(1 \mathrm{~mm}), 0.7 \%(2 \mathrm{~mm})$ and $5 \%(3 \mathrm{~mm})$. Table 1 shows that when this is combined with the shift of the reflectivity null from $10 \mathrm{GHz}$, which is up to $2 \%, 5 \%$ and $9 \%$ respectively, the common reflectivity bandwidth of the absorber working at $40^{\circ}$ and $50^{\circ}$ incidence is reduced from the maximum value obtained for all three structures at the nominal design angle $\left(45^{\circ}\right)$. The numerical results also show that the FSS based absorbers can operate independently of the polarization of the incident waves over the entire angular range from $40^{\circ}$ to $50^{\circ}$, yielding $-10 \mathrm{~dB}$ TE/TM reflectivity bandwidths of $1.3 \%(1 \mathrm{~mm}), 5.7 \%(2 \mathrm{~mm})$ and $17.7 \%(3 \mathrm{~mm})$.

\section{CONSTRUCTION AND EXPERIMENTAL RESULTS}

A $15 \times 15 \mathrm{~cm}^{2}$ metal backed $3 \mathrm{~mm}$ thick FSS absorber was fabricated and measured to experimentally to validate the design methodology and simulation results presented in the previous section. The FSS composed of a doubly periodic array with $26 \times 14$ unit cells was printed on a $0.13 \mathrm{~mm}$ thick Taconic TLY-5 microwave laminate $\left(\varepsilon_{\mathrm{r}}=2.2, \tan \delta=0.0009\right)$ and bonded to a $3 \mathrm{~mm}$ thick metal backed Rhoacell foam spacer to form a HIS. Physical dimensions of twenty unit cells were measured and the tolerances on the side lengths were found to be within $\pm 2 \%$ of the nominal design parameters. In order to avoid parasitic impedances and high component and manufacturing costs, instead of lumped resistors loading the rectangular loop elements, thin layers of electro-conductive shielding paint, Y Shield HSF - 74 [14], were deposited across the $0.7 \mathrm{~mm}\left(w_{T E}\right)$ and $2.05 \mathrm{~mm}\left(w_{T M}\right)$ wide gaps in the horizontal and vertical side arms of the printed loops. The ink is a graphite and carbon black based material, which is reinforced with long conductive fibers, providing up to $36 \mathrm{~dB}$ signal attenuation at frequencies up to $18 \mathrm{GHz}$ when applied at a coverage rate of $7.5 \mathrm{~m}^{2} / \mathrm{I}$ [14]. Although the painted ink traces are inhomogeneous, the total resistance is proportional to the trace length and inversely proportional to the ink thickness [2], [15], [16]. The numerical estimates show that the 
optimum performance is obtained when the gaps in the vertical and horizontal arms of the loops are filled with elements with surface resistance values of $175 \Omega$ /square and $111 \Omega$ /square respectively (Figure 1). In order to obtain these values for the prototype absorber, an experimental investigation was carried out to establish the relationship between the thickness and the surface resistance of the Y Shield HSF - 74 shielding paint. A $15 \times 15 \mathrm{~cm}^{2}$ array of linear dipoles with nominal length $9.9 \mathrm{~mm}$ and width $1.6 \mathrm{~mm}$, was patterned on a $0.13 \mathrm{~mm}$ Taconic TLY-5 substrate using a $50 \mu \mathrm{m}$ thick Kapton film stencil. Physical measurements of the average thickness of the dipoles constructed of 1 to 4 layers of ink $(21.9,33.8,43.7,55.5 \mu \mathrm{m})$ were obtained using a Tencor Alpha-Step 200 Profilometer. The four spectral transmission responses were measured at normal incidence over the frequency range $9-18 \mathrm{GHz}$, allowing 24 hours for each ink layer to cure before the experimental data acquisition. CST predictions were compared to the measured transmission coefficients, and the surface resistance value used in the numerical model was varied to obtain the best fit as shown in Figure 5. The relationship between ink thickness and surface resistance is summarized in Figure 6, and was used to determine the number of ink layers needed to obtain values close to the required resistive loading. The graph shows that the surface resistance is inversely proportional to the ink thickness, and for the $3 \mathrm{~mm}$ thick structure only 1 layer of HSF-74 is sufficient to form the two different resistive elements. Conversely several layers of shielding paint would need to be applied to obtain the much smaller vertical and horizontal resistance values that are required to construct the 1 $\mathrm{mm}$ and $2 \mathrm{~mm}$ thick absorbers shown in Figure 1, and therefore these would be more difficult to manufacture. The ink was applied manually using a stencil and it was found that the applied coating was thinner than the single layer FSS test structure used to obtain the results depicted in Figure 6. Therefore to reduce the surface resistances a second layer of ink was deposited in the unequal size horizontal and vertical loop gaps in $90 \%$ of the unit cells. Measurements of 38 samples yielded average ink thickness of $17 \mu \mathrm{m}$ and $24 \mu \mathrm{m}$ respectively, corresponding approximately to surface resistances of $210 \Omega$ /square and $130 \Omega$ /square (extrapolated from Figure 6) , which is close to the desired values of $175 \Omega /$ square and $111 \Omega$ /square. A photograph of 4 of the 364 unit cells of the 
periodic array and the bistatic set-up used to measure the radar backscatter in the TE and TM plane is shown in Figure 7. The time gated reflection measurements were performed in the frequency range $7-14 \mathrm{GHz}$ using a pair of standard gain horns positioned a distance $47 \mathrm{~cm}$ from the $15 \times 15$ $\mathrm{cm}^{2}$ sample. A metal plate of the same size was first used to calibrate the experimental set-up, and then substituted with the metal backed resistive FSS. In Figure 8(a) the measured TE and TM plane reflectivity plots at $45^{\circ}$ incidence are compared to the CST simulations using the retrieved resistances $\approx 210 \Omega /$ square and $\approx 130 \Omega /$ square and the optimum surface resistance values 175 $\Omega /$ square and $111 \Omega /$ square. Very good agreement between the measurements and simulations is observed, and moreover, the measured resonance frequencies of the absorber, coincident for both polarizations, are shifted upwards only by $5 \%$ as compared with the optimum computed backscatter response, even though the surface resistance values are $20 \%$ larger. Resistances closer to the specified values may be realized if the ink thickness is carefully controlled using a more precise deposition method. Our investigation has shown that a further incremental increase in the ink thickness by the simple stencil printing technique would degrade the absorber performance.

Backscatter from the absorber was also measured at $40^{\circ}$ and $50^{\circ}$ incidence in the TE and TM plane. The results obtained in the TM plane are similar to the computed responses depicted in Figure 3 and confirm that the reflection coefficients are reasonably sensitive to the variation in the angle of incidence of the absorber about $45^{\circ}$. For brevity only the comparison between the measured and predicted TE plane reflectivity responses is plotted in Figure $8(\mathrm{~b})$ which demonstrates close correlation and better angular stability over the $40^{\circ}$ to $50^{\circ}$ range. 


\section{CONCLUSIONS}

In this paper we investigated the feasibility of designing a thin metal backed single layer FSS array which operates as a microwave absorber for TE and TM polarized waves at large angles of incidence, and demonstrated the principle for a tilt angle of $45^{\circ}$. Independent and controllable backscatter suppression in the orthogonal planes is obtained by creating an array of asymmetrical split loop elements which are impedance matched to free space by loading the gaps with resistor pairs of unequal resistance. The numerical results and design methodology have been validated by experimental data acquired with a bistatic set-up for a $3 \mathrm{~mm}$ thick absorber illuminated at angles $45^{\circ}$. Although the absorber is shown to work over a wide range of angles centered at $45^{\circ}$ for $\mathrm{TE}$ waves, this is not the case for TM polarisation, and therefore for applications that require a wider angular range, well known design techniques to reduce the unit cell type can be implemented. An important outcome of this work is the demonstration of the use of high frequency paint [14], normally applied to interior or exterior walls of buildings to provide electromagnetic shielding, as a means to provide the small surface resistances that are required to optimize the performance of this class of thin FSS absorbers.

\section{ACKNOWLEDGEMENT}

S. N. Zabri is supported by a research scholarship from University Teknikal Malaysia Melaka, Durian Tunggal, Malaysia 


\section{REFERENCES}

[1] Chambers B.: 'Optimum design of a Salisbury screen radar absorber', Electron. Lett., 1994, 30, (16), pp. 1353-1354

[2] Che Seman, F., Cahill, R., Fusco, V. F., Goussetis, G.: 'Design of a Salisbury screen absorber using frequency selective surfaces to improve bandwidth and angular stability performance', IET Microwaves, Antennas Propag., 2011, 5, (2), pp. 149-156

[3] Yang, J., Shen, Z.: 'A Thin and Broadband Absorber Using Double-Square Loops', Antennas Wirel. Propag. Lett., 2007, 6, (11), pp. 388-391

[4] Costa, F., Monorchio, A., Manara, G.: 'Analysis and Design of Ultra Thin Electromagnetic Absorbers Comprising Resistively Loaded High Impedance Surfaces', IEEE Trans. Antennas Propag., 2010, 58, (5), pp. 1551-1558

[5] Li, Y., Fu, Y., Yuan, N.: 'Characteristics estimation for high-impedance surfaces based ultrathin radar absorber', Microw. Opt. Technol. Lett., 2009, 51, (7), pp. 1775-1778

[6] Dickie, R., Cahill, R., H. S. Gamble, et al.: 'Polarisation independent bandpass FSS', Electron. Lett., 2007, 43, (19), pp. 1013-1015

[7] Dickie, R., Cahill, R., Gamble, H., et al.: 'Submillimeter Wave Frequency Selective Surface With Polarization Independent Spectral Responses', IEEE Trans. Antennas Propag., 2009, 57, (7), pp. 1985-1994

[8] Zabri, S. N., Cahill, R., Schuchinsky, A.: 'Polarisation independent split ring frequency selective surface', Electron. Lett., 2013, 49, (4), pp. 245-246

[9] Munk, B. A.: 'Frequency Selective Surfaces: Theory and Design' ( John Wiley \& Sons, 2000)

[10] Dickie, R., Cahill, R., Mitchell, N., et al.: '664 GHz dual polarisation frequency selective surface', Electron. Lett., 2010, 46, (7), pp. 472-474

[11] Chuprin, A. D., Parker, E. A., Batchelor, J. C.: 'Resonant frequencies of open and closed loop frequency selective surface arrays', Electron. Lett., 2000, 36, (19), pp. 1601-1603

[12] 'CST - Computer Simulation Technology', https://www.cst.com/, accessed January 2013 
[13] Pozar, D. M., Targonski, S. D., Syrigos, H. D.: 'Design of millimeter wave microstrip reflectarrays', IEEE Trans. Antennas Propag., 1997, 45, (2), pp. 287-296

[14] 'Y-SHIELD EMR Protection', http://www.yshield.com/, accessed January 2013

[15] Che Seman, F., Cahill, R.: 'Performance enhancement of Salisbury screen absorber using resistively loaded spiral FSS', Microw. Opt. Technol. Lett., 2011, 53, (7), pp. 1538-1541

[16] Sidén, J., Fein, M. K., Koptyug, A., Nilsson, H.-E.: 'Printed antennas with variable conductive ink layer thickness', IET Microwaves, Antennas Propag., 2007, 1, (2), pp. 401-407 


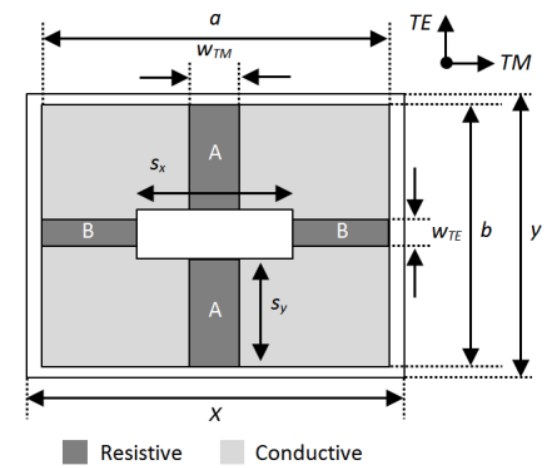

(a)

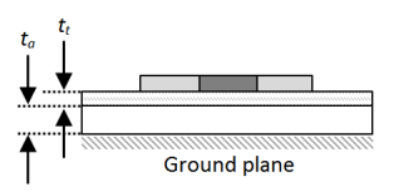

(b)

Figure 1 Schematic plot of the unit cell geometry and dimensions of the FSS absorber, (a) top view, (b) side view

$\underline{t}_{a}=1 \mathrm{~mm}, x=13.4 \mathrm{~mm}, y=10.4, a=12 \mathrm{~mm}, b=9.4 \mathrm{~mm}, s_{x}=5 \mathrm{~mm}, s_{y}=3.7 \mathrm{~mm}, w_{T E}=1 \mathrm{~mm}, w_{T M}=$ $2.05 \mathrm{~mm}, R_{T E}=18 \Omega / \square, R_{T M}=13 \Omega / \square, t_{t}=0.13 \mathrm{~mm}$

$\underline{t}_{a}=2 \mathrm{~mm}, x=11.9 \mathrm{~mm}, y=7.4, a=10.7 \mathrm{~mm}, b=6.6 \mathrm{~mm}, s_{x}=5 \mathrm{~mm}, s_{y}=1.98 \mathrm{~mm}, w_{T E}=1 \mathrm{~mm}, w_{T M}=$ $2.05 \mathrm{~mm}, R_{T E}=54 \Omega / \square, R_{T M}=46 \Omega / \square, t_{t}=0.13 \mathrm{~mm}$

$\underline{t}_{\underline{a}}=3 \mathrm{~mm}, x=11.1 \mathrm{~mm}, y=5.9, a=10 \mathrm{~mm}, b=5.3 \mathrm{~mm}, s_{x}=4.2 \mathrm{~mm}, s_{y}=2.1 \mathrm{~mm}, w_{T E}=0.7 \mathrm{~mm}, w_{T M}=$ $2.05 \mathrm{~mm}, R_{T E}=175 \Omega / \square, R_{T M}=111 \Omega / \square, t_{t}=0.13 \mathrm{~mm}$ 


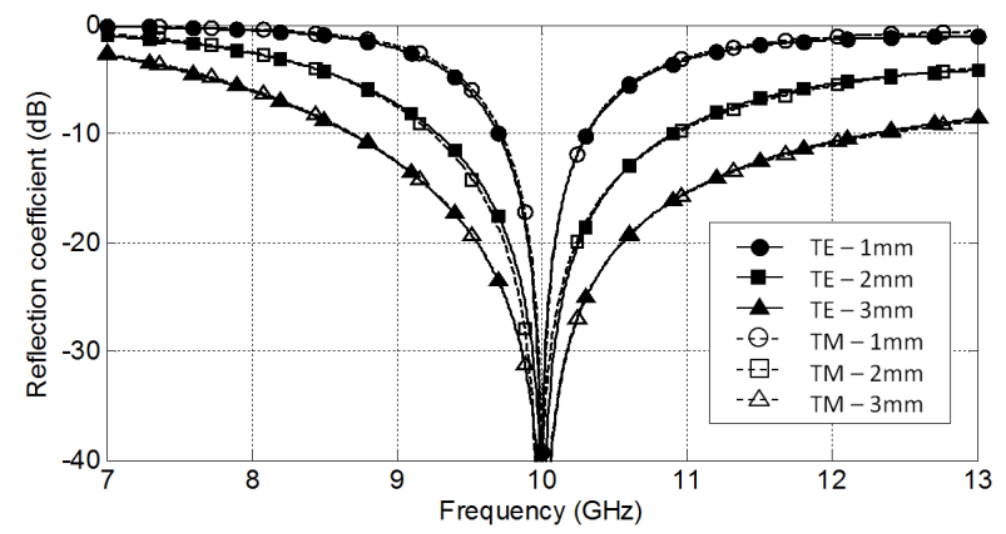

Figure 2 Computed reflectivity plots for $1 \mathrm{~mm}, 2 \mathrm{~mm}$ and $3 \mathrm{~mm}$ thick absorbers for TE and TM polarizations $-45^{\circ}$ incidence 


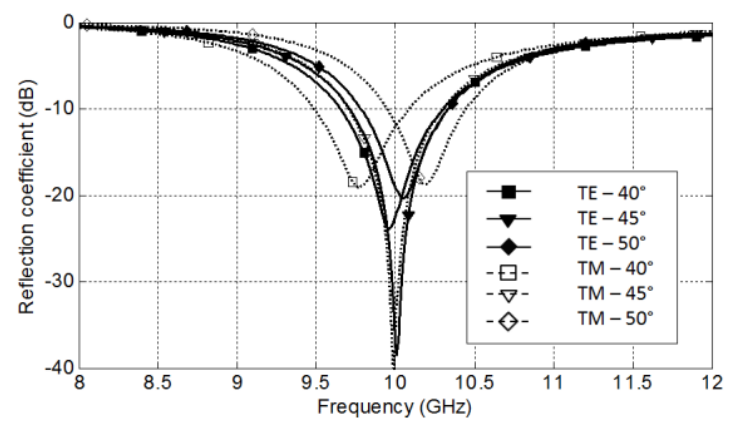

(a)

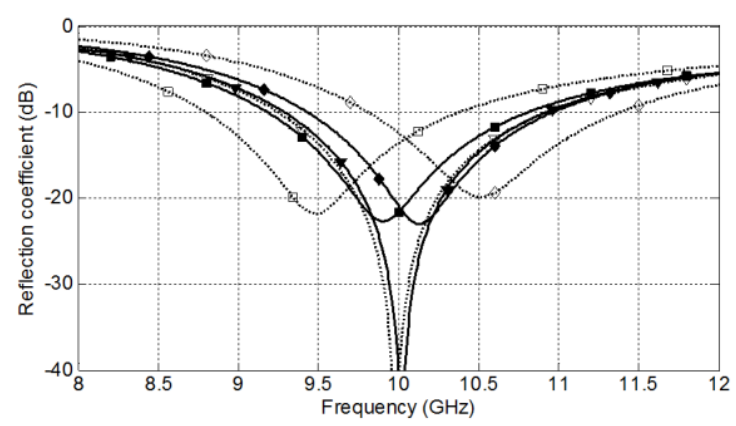

(b)

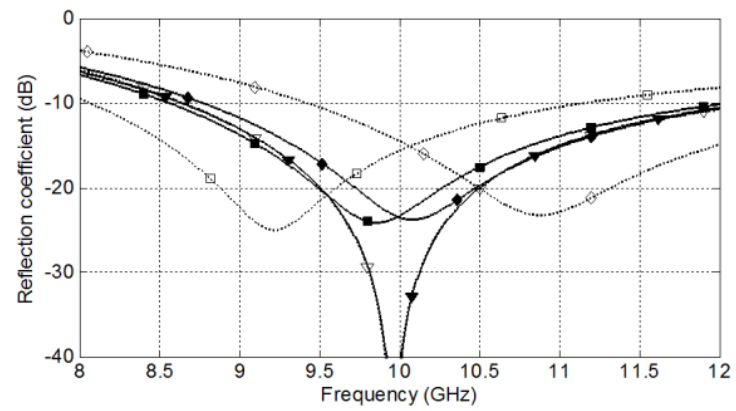

(c)

Figure 3 Computed reflectivity plots for TE and TM polarizations at $40^{\circ}, 45^{\circ}$ and $50^{\circ}$ incidence and absorber thicknesses - a) $1 \mathrm{~mm}$, b) $2 \mathrm{~mm}$ and c) $3 \mathrm{~mm}$ 


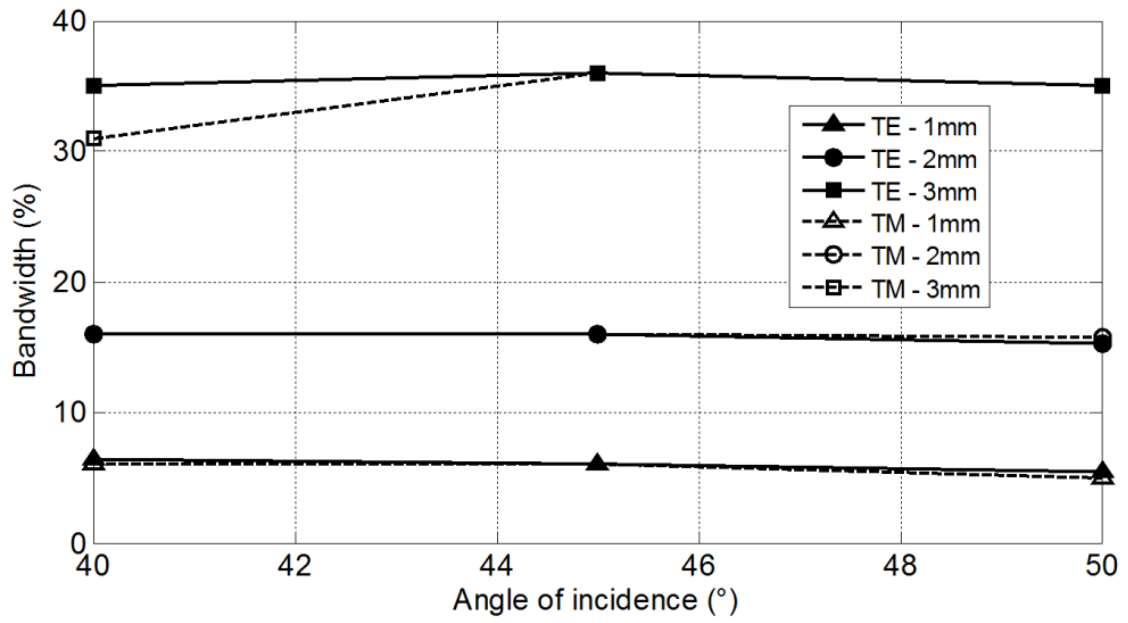

Figure 4 Predicted $-10 \mathrm{~dB}$ bandwidths of FSS absorbers as a function of thickness for TE and TM polarized waves at $40^{\circ}, 45^{\circ}$ and $50^{\circ}$ incidence. 


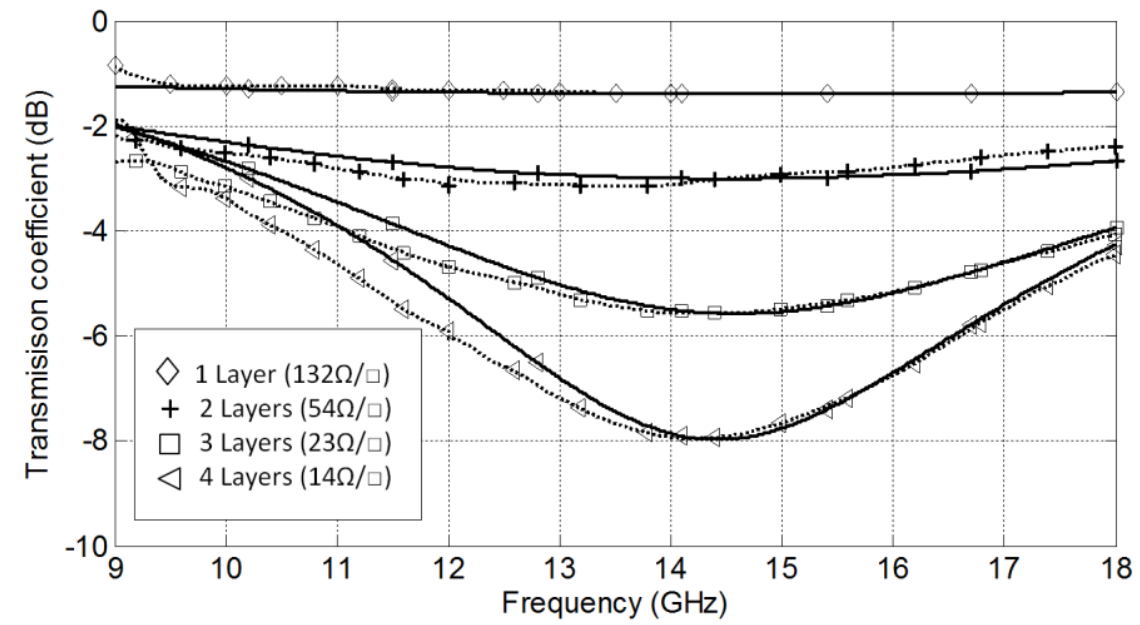

Figure 5 Computed (solid lines) and measured (dotted lines) transmission response of 1, 2, 3 and 4 layer dipole FSS constructed of HSF-74 shielding paint 


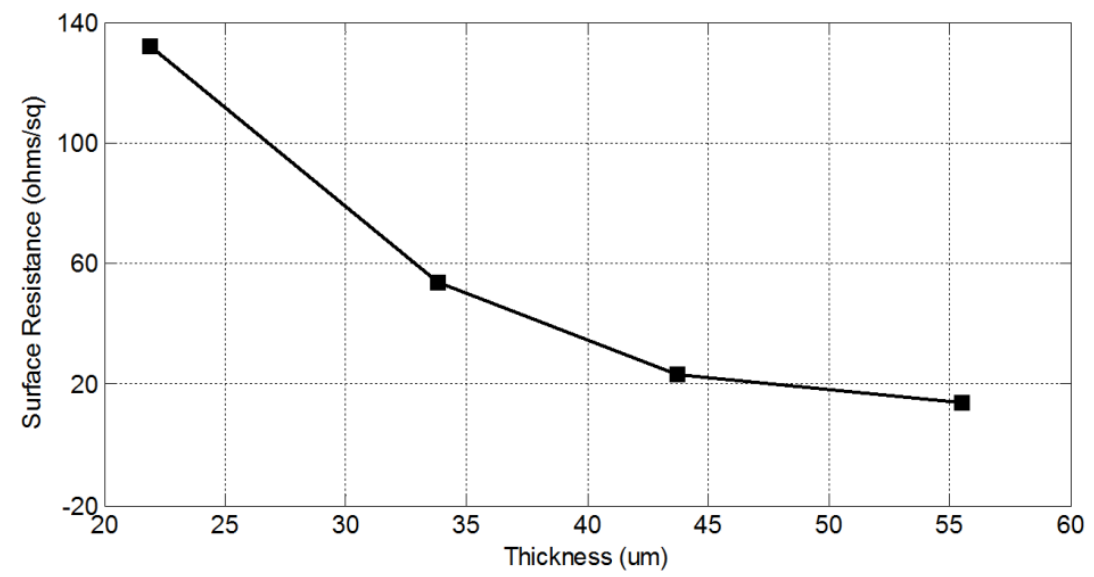

Figure 6 Measured relationship between surface resistance and thickness for HSF-74 ink 

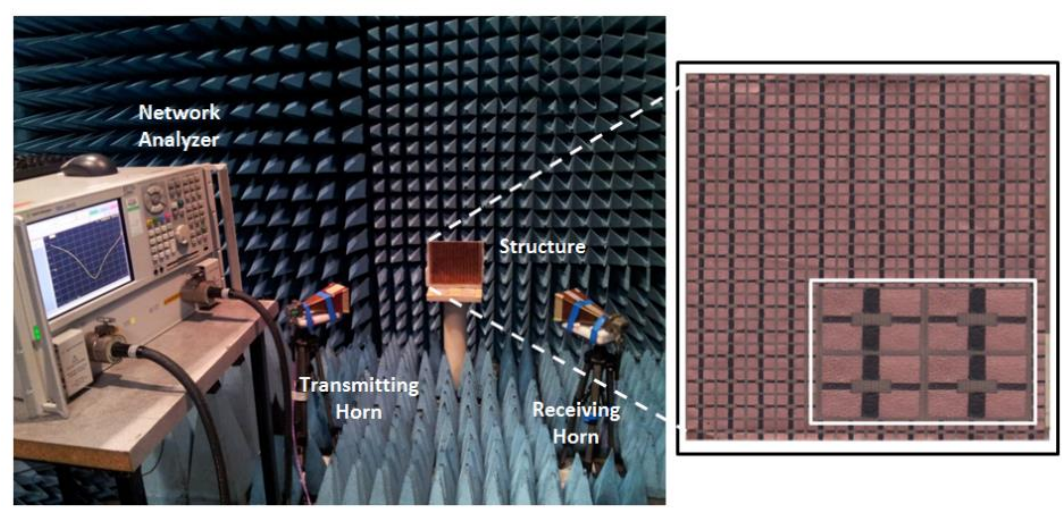

Figure 7 Photograph of a $26 \times 14$ array of resistively loaded FSS loop elements and bistatic reflectivity measurement set-up 

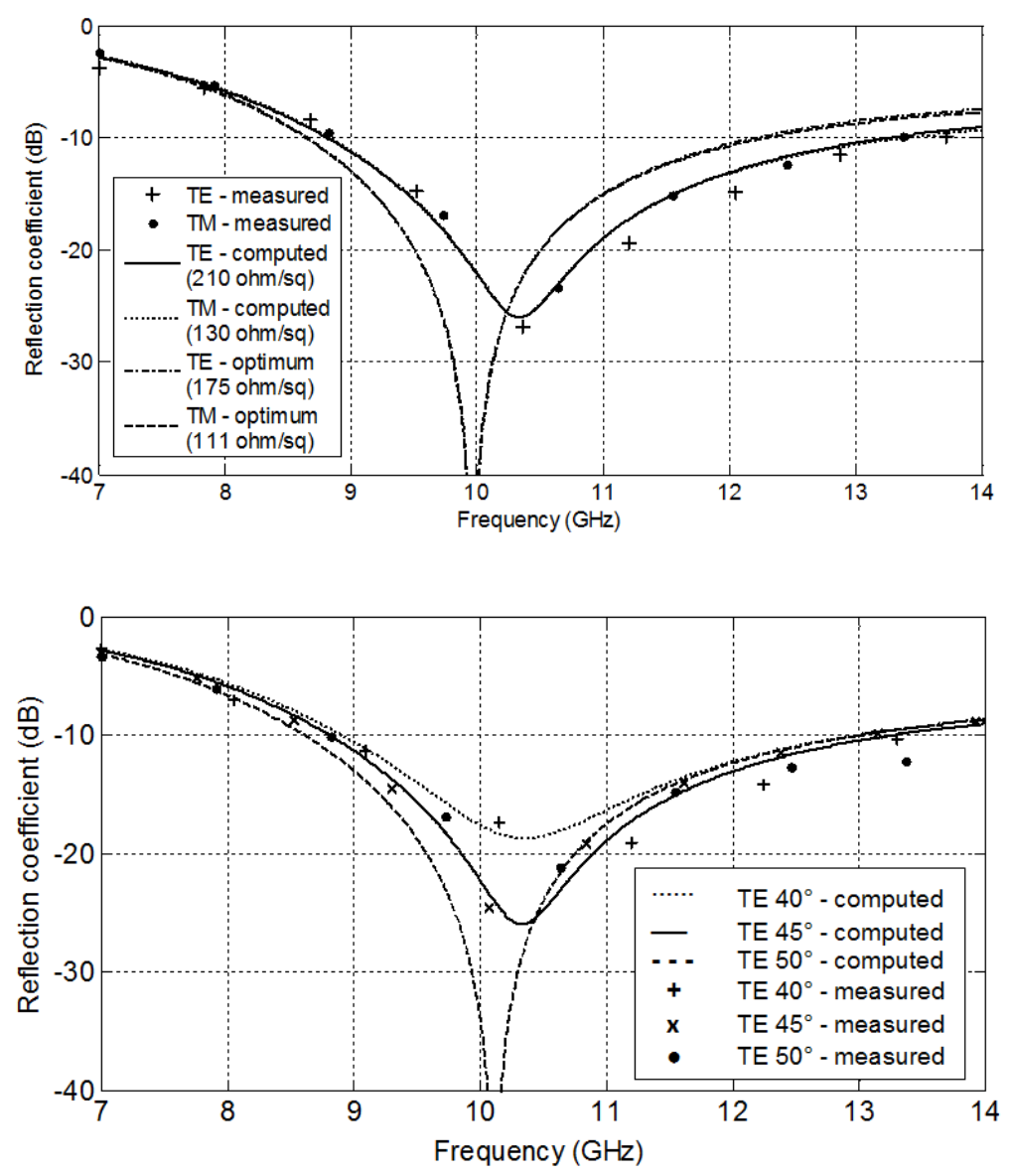

Figure 8 Measured and computed reflectivity plots for $3 \mathrm{~mm}$ thick FSS absorber for TE and TM polarizations a) TE and TM plane at $45^{\circ}$ b) TE plane at $40^{\circ}$ to $50^{\circ}$ 
Table 1 Computed common (TE/TM) -10 dB reflectivity bandwidth for $1 \mathrm{~mm}, 2 \mathrm{~mm}$ and $3 \mathrm{~mm}$ thick FSS absorber operating at $40^{\circ}, 45^{\circ}$ and $50^{\circ}$.

\begin{tabular}{|c|c|c|c|}
\hline & $1 \mathrm{~mm}$ & $2 \mathrm{~mm}$ & $3 \mathrm{~mm}$ \\
\hline $40^{\circ}$ & $3.9 \%$ & $11 \%$ & $26 \%$ \\
\hline $45^{\circ}$ & $6 \%$ & $16 \%$ & $36 \%$ \\
\hline $50^{\circ}$ & $3.6 \%$ & $11 \%$ & $28 \%$ \\
\hline
\end{tabular}

